\title{
Nervenverletzungen als Ursache nachweisbar
}

Nach Knieoperationen leiden Patienten immer wieder an heftigen chronischen Schmerzen. Deren Ursache wurde zwar in der Schädigung von kleinen Nerven vermutet, war jedoch lange nicht bildgebend nachweisbar.

Nach einer Operation am Kniegelenk kann es, trotz erfolgreicher Operation, immer wieder zu monatelangen, chronischen und unklaren Schmerzen kommen. Bei einem Großteil der Patienten bleiben diese Schmerzen erträglich und verschwinden schließlich von alleine wieder. Fünf bis zehn Prozent leiden jedoch ein- bis eineinhalb Jahre später immer noch an teils starken neuropathischen Schmerzen. Wenn keine anderen Ursachen feststellbar sind, ist dies der erste Hinweis, dass eine Nervenverletzung die Ursache sein könnte.

Bei chirurgischen Eingriffen sind Verletzungen von Gewebe und Nerven im operierten Areal nicht zu vermeiden. Meistens bleiben diese Schädigungen ohne weitere Folgen. Wenn ein Nerv allerdings so geschädigt ist, dass die Schmerzen auch Monate nach der Operation noch anhalten oder sogar stärker werden, sollte so rasch wie möglich mit einer gezielten Schmerztherapie begonnen werden, um eine Chronifizierung des Schmerzes zu verhindern.

Bisher war es allerdings nicht möglich, die weniger als einen Millimeter dünnen Hautnerven um das Knie und deren Verästelungen in bildgebenden Verfahren sichtbar zu machen. Somit konnte eine Nervenverletzung immer nur vermutet und auf Verdacht behandelt werden. Ist die Vermutung aber falsch, kann so eine Therapie eher schaden als nützen. Nun konnte ein interdisziplinäres Team um den Radiologen Dr. Georg Riegler von der Universitätsklinik für Radiologie und Nuklearmedizin der MedUni Wien, in Kooperation mit dem PUC - Private Ultrasound Center- sowie der Klinischen Abteilung für Plastische und
Rekonstruktive Chirurgie und dem Zentrum für Anatomie und Zellbiologie der MedUni Wien, diese kleinen Hautnerven am Knie erstmals mittels hochauflösendem Ultraschall sichtbar machen $[1,2]$.

\section{Gezielte Therapie ermöglichen} In der ersten Studie des Teams gelang es, den Ramus infrapatellaris (ein sensibler Nervenast vor und unterhalb der Kniescheibe) über seinen gesamten Verlauf mit Hilfe des hochauflösenden Ultraschalls darzustellen. In der zweiten Studie konnten die verzweigten vorderen Hautäste des Oberschenkelnervs Nervus femoralis dargestellt werden. Es konnte auch gezeigt werden, dass diese Nervenbahnen hoch variabel sind und bei jedem Menschen anders verlaufen. Es ist also unerlässlich, die genaue Position der verletzten Nervenfasern zu bestimmen, bevor eine gezielte Therapie gegen diese Nervenschmerzen angewandt werden kann.

\section{Exakte Lokalisation des Schmerzes} Für die genaue Eingrenzung der Schmerz verursachenden Nervenäste sei es laut Riegler unbedingt erforderlich, eine "diagnostische Blockade" vorzunehmen: „Da die Nervenversorgung so variabel ist, muss zuerst sichergestellt werden, welcher dieser winzigen Nerven die Schmerzen verursacht. Dies kann nur durch eine selektive vorübergehende Betäubung, also einer Blockade des verdächtigten Nervs, mit maximal einem Milliliter Betäubungsmittel erreicht werden. Wenn der Schmerz sich direkt nach erfolgter Blockade deutlich reduziert und nach Abklingen der Betäubung zurückkehrt, dann ist das Problem lokalisiert".
Ist ein Nerv Ursache für die Schmerzen, gibt es verschiedene therapeutische Möglichkeiten:Zuerst wird eine lokale Therapie mittels Schmerzpflastern oder eine Physiotherapie versucht. Die nächste Stufe wäre eine ultraschallgezielte Therapie, bei der in einigen Sitzungen der betroffene Nerv mehrmals mittels Anästhetikums blockiert wird und somit der Schmerz gehemmt wird. Außerdem sind Kortison-Gaben und eine Radiofrequenzablation anzudenken. Erst als letzte Option werden chirurgische Verfahren wie die Freilegung oder Durchtrennung des Nervs durchgeführt, dies aber bereits mit guten Erfolgen.

Riegler:„Es ist sehr wichtig, so rasch wie möglich eine richtige Diagnose zu haben und nicht zu lang zu warten. Die Schmerzen können sich sonst in das ,Schmerzgedächtnis einbrennen' und chronisch werden".

\footnotetext{
Literatur

1. Pivec C et al (2018) Novel Demonstration of the Anterior Femoral Cutaneous Nerves using Ultrasound. Ultraschall Med. https:// doi.org/10.1055/s-0043-121628

2. Riegler G. et al (2018) Ultrasound Anatomic Demonstration of the Infrapatellar Nerve Branches. Arthroscopy 34(10):2874-2883. https://doi.org/10.1016/j.arthro.2018.05.043
}

rheuma plus $2019 \cdot 18: 52$ https://doi.org/10.1007/s12688 019-0240-4

(c) Springer-Verlag GmbH Austria, ein Teil von Springer Nature 2019 